\title{
Human retinal pigment epithelial cells are protected against hypoxia by BNIP3
}

\author{
Jingyang Feng ${ }^{1,2 \#}$, Wei Tan ${ }^{3 \#}$, Tong Li $^{1,2}$, Quan Yan $^{1,2}$, Hong Zhu ${ }^{1,2,4}$, Xiaodong Sun ${ }^{1,2,4}$ \\ ${ }^{1}$ Department of Ophthalmology, Shanghai General Hospital (Shanghai First People's Hospital), Shanghai Jiao Tong University, School of Medicine, \\ Shanghai, China; ${ }^{2}$ Shanghai Engineering Center for Visual Science and Photomedicine, Shanghai, China; ${ }^{3}$ Department of Ophthalmology, Zunyi \\ First People's Hospital, The Third Affiliated Hospital of Zunyi Medical University, Zunyi, China; ${ }^{4}$ Shanghai Key Laboratory of Fundus Diseases, \\ Shanghai, China \\ Contributions: (I) Conception and design: J Feng, H Zhu; (II) Administrative support: X Sun; (III) Provision of study materials or patients: Q Yan; (IV) \\ Collection and assembly of data: J Feng, W Tan, T Li; (V) Data analysis and interpretation: J Feng, W Tan; (VI) Manuscript writing: All authors; (VII) \\ Final approval of manuscript: All authors. \\ \#These authors contributed equally to this work. \\ Correspondence to: Hong Zhu, MD, PhD. Department of Ophthalmology, Shanghai General Hospital (Shanghai First People's Hospital), Shanghai \\ Jiao Tong University, School of Medicine, Shanghai 200030, China. Email: zhuhongchina@126.com.
}

Background: Hypoxia has been implicated in the process of retinal pigment epithelium (RPE) dysfunction. However, recent studies suggest that hypoxia contributes to survival rather than cell death through induction of Bcl-2/adenovirus E1B 19-kDa interacting protein 3 (BNIP3)-dependent autophagy. In contrast, persistent oxidative stress was found to result in autophagy dysregulation in RPE cells. These seemingly contradictory findings led us to investigate the potential role of BNIP3, a crucial mediator of hypoxia-induced autophagy, in the context of hypoxic RPE cells.

Methods: Human RPE D407 cells were treated with low-oxygen conditions, and cell growth, apoptosis, and autophagy was assessed by Cell Counting Kit-8 assay, flow cytometry analysis and immunofluorescence staining, respectively.

Results: Hypoxic conditions simultaneously triggered a large amount of apoptosis and inhibited autophagy. Moreover, hypoxia led to severe impairments, including the stimulation of reactive oxygen species, and reduction of mitochondrial membrane potential, and adenosine triphosphate production. The stimulation of autophagy by rapamycin inhibited hypoxia-induced severe impairments to a great extent. Interestingly, similar results were observed for BNIP3 overexpression, which can be largely blocked by 3-MA, a welldefined inhibitor of autophagy. Moreover, BNIP3 knockdown further aggravated hypoxia-induced impairments in D407 cells, which can be reversed by rapamycin.

Conclusions: Collectively, these results indicated that BNIP3 can protect human retinal pigmented epithelial cells under hypoxic conditions by inducing autophagy.

Keywords: Retinal pigment epithelium (RPE); hypoxia; apoptosis; autophagy; Bcl-2/adenovirus E1B 19-kDa interacting protein 3 (BNIP3)

Submitted Sep 23, 2020. Accepted for publication Nov 13, 2020.

doi: $10.21037 /$ atm-20-7145

View this article at: http://dx.doi.org/10.21037/atm-20-7145 


\section{Introduction}

Age-related macular degeneration (AMD) is a predominant cause of severe and irreversible loss of vision in elderly people worldwide $(1,2)$. Generally, AMD begins in the retinal pigment epithelium (RPE) which serve as a passthrough between the retina and choroid (3). The RPE cells have a variety of important functions, all of which can influence AMD disease (4). Degeneration of RPE cells can result in catastrophic effects to the vision (5). Currently, it has been recognized that age-related change in RPE cells is one of the early hallmarks of AMD pathogenesis $(4,6,7)$.

Previous studies have suggested that hypoxia in RPE cells may be a central risk factor for AMD (5). The hypoxic state enables the production of a large amount of reactive oxygen species (ROS) by the electron transport chain (ETC), which can severely damage the cells (8). Hypoxia is a natural consequence of aging microenvironments and serves as essential stimulus to numerous age-related diseases (5), including the progression of AMD $(9,10)$. Our understanding of hypoxia was greatly altered by the recent work of Bellot et al., that revealed hypoxia (0.1-3\% oxygen) contributed to survival as opposed to death in various cell types by evoking an adaptive mechanism of autophagy, also known as mitochondrial autophagy $(11,12)$. However, in terms of RPE cells, it seems contradictory to the observations of Mitter et al., who found persistent oxidative stress resulted in the dysregulation of autophagy, which increased the susceptibility to AMD (13).

These seeming contradictions motivated us to follow the steps of previous work. According to Bellot et al., the Bcl-2/ adenovirus E1B 19-kDa interacting protein 3 (BNIP3) plays an essential role in hypoxia-induced mitochondrial autophagy, which was known to serve a protective role in a variety of normal and cancerous cells when subjected to hypoxic conditions (14). This process requires the hypoxia-inducible factor 1 (HIF-1)-dependent expression of BNIP3 and the constitutive expression of Beclin-1 and autophagy-related 5 (Atg5) (12), which together form the important signaling cascade in the regulation of autophagy that is the HIF-1/ BNIP3/Beclin-1 pathway (15). To the best of our knowledge, no study has yet reported on the potential role of BNIP3 in the context of hypoxic assaults on human RPE cells.

In the present study, we treated D407 cells with $1 \%$ oxygen and found the hypoxic microenvironment significantly activated apoptosis and inhibited autophagy simultaneously. Moreover, hypoxia resulted in severe impairments, including the stimulation of ROS, reduction of mitochondrial membrane potential (MMP), and adenosine triphosphate (ATP) production. Notably, all of these effects could be counteracted by the activation of autophagy with both rapamycin and BNIP3 overexpression. Further studies confirmed that BNIP3 can protect human retinal pigmented epithelial cells under hypoxic conditions by inducing BNIP3/Beclin/Atg-mediated autophagy.

We present the following article in accordance with the MDAR reporting checklist (available at http://dx.doi. org/10.21037/atm-20-7145).

\section{Methods}

\section{Cell culture and bypoxic treatment}

The human RPE cell line (D407) was purchased from Cell Bank, Shanghai Institutes for Biological Sciences, Chinese Academy of Sciences (Shanghai, China). The D407 cells were cultured in Roswell Park Memorial Institute (RPMI) 1640 medium supplemented with $10 \%$ fetal bovine serum (FBS) and 1\% Penicillin-Streptomycin solution (Thermo Fisher Scientific, Inc., Waltham, MA, USA) in $5 \% \mathrm{CO}_{2}$ at $37^{\circ} \mathrm{C}$. To create hypoxic conditions, the oxygen level was maintained at $1 \%$.

\section{Vector construction}

The BNIP3 overexpression vector was constructed by cloning the complementary (c)DNAs of human BNIP3 into a pLVX-Puro vector (Clontech, Mountain View, CA, USA) using EcoRI and BamHI restriction sites. Short hairpin (sh)RNAs designed to target the sequence (CCTGGGTAGAACTGCACTT) of the BNIP3 transcript were cloned into the lentiviral shRNA vector pLKO.1. The forward sequence was 5'-CCGGCCTGGGTAGAACTG CACTTCTCGAGAAGTGCAGTTCTACCCAGGTTT TT-3', and the reverse sequence was 5'-AATTAAAAACC TGGGTAGAACTGCACTTCTCGAGAAGTGCAGTT CTACCCAGG-3'. All of the constructs were confirmed by sequencing.

\section{Gene overexpression and knockdown}

Knockdown of BNIP3 was with a pLKO based lentiviral vector and $\mathrm{pLVX}$-Puro retroviral vectors were used for overexpression of BNIP3. Lentiviruses were generated in human embryonic kidney (HEK) 293T cells as described (16), and then D407 cells were infected with virus-containing supernatants. The alteration of expression of BNIP3 was confirmed by real-time quantitative reverse transcription- 
polymerase chain reaction (qRT-PCR) and western blot analysis.

\section{Cell proliferation assay}

Cell Counting Kit-8 (CCK-8, SAB Biotech, College Park, $\mathrm{MD}$, USA) was used to evaluate cell viability according to the manufacturer's instructions. The D407 cells were seeded into 96-well plates $\left(3 \times 10^{3}\right.$ cells/well). At different time points $(0,24,48$, and $72 \mathrm{~h})$, the medium was changed to $100 \mu \mathrm{L}$ cell culture medium containing CCK-8 (volume ratio of RPMI 1640 medium to CCK-8 was 9:1) for each well. Afterwards, cells were incubated at $37^{\circ} \mathrm{C}$ for $1 \mathrm{~h}$, and then the absorbance at $450 \mathrm{~nm}$ was detected using a microplate reader.

\section{Apoptosis assay}

Annexin V-FITC Apoptosis Detection Kit (Beyotime Institute of Biotechnology, Beijing, China) was used to evaluate cell apoptosis in accordance with the manufacturer's instructions. Specifically, $1 \times 10^{6}$ of D407 cells were collected, washed, and resuspended in $195 \mu \mathrm{L}$ Annexin V-FITC binding buffer. Then, the samples were contained with Annexin V-FITC $(5 \mu \mathrm{L})$ and propidium iodide PI $(5 \mu \mathrm{L})$ in the dark for $15 \mathrm{~min}$. Next, apoptotic cells were determined by flow cytometry.

\section{Immunofluorescence assay}

Immunofluorescence assay was carried out as previously reported (17). Briefly, cells on round glass coverslips was fixed with prechilled methanol, permeabilized with phosphate-buffered saline (PBS) containing 0.2\% Triton X, and blocked with PBS supplemented with $1 \%$ bovine serum albumin (BSA). Next, the coverslips were incubated with anti-LC3B antibody (Abcam, Cambridge, MA, USA) at $4{ }^{\circ} \mathrm{C}$ overnight. After washing with PBS, coverslips were then incubated with Alexa Fluor 488-labeled Goat Anti-Rabbit $\operatorname{IgG}(\mathrm{H}+\mathrm{L})$ as the secondary antibody for $1 \mathrm{~h}$ in darkness. The coverslips were then washed completely and mounted onto glass slides with 4',6-diamidino-2-phenylindole (DAPI) containing mounting medium.

\section{ROS assay}

The generation of ROS was measured using a DCFH-DA fluorescence probe (Beyotime Institute of Biotechnology,
Beijing, China). After being incubated with DCFH-DA $(10 \mu \mathrm{M})$ for $20 \mathrm{~min}$ at $37^{\circ} \mathrm{C}$ in a humidified atmosphere in the dark, cells were analyzed with flow cytometry using excitation at $480 \mathrm{~nm}$ and emission at $525 \mathrm{~nm}$.

\section{Measurement of MMP}

MMP was measured with flow cytometry as previously reported (18). Briefly, cells were collected and stained with JC-1 (Beyotime Institute of Biotechnology, Beijing, China) for $20 \mathrm{~min}$ at $37^{\circ} \mathrm{C}$. After washing to remove extracellular JC-1, measurements were performed using flow cytometry.

\section{Measurements of intracellular ATP levels}

Levels of ATP were determined by colorimetric method using the ATP assay kit (Nanjing Jiancheng Bioengineering Institute, Nanjing, China) according to the manual. Briefly, cells were collected and homogenized. After centrifugation, the supernatants were collected and the protein concentration measured with bicinchoninic acid (BCA) reagent (Thermo Fisher Scientific, Inc., Waltham, MA, USA). The final ATP concentration of each sample was normalized to protein concentration and was reported in terms of mmol/g cellular protein.

\section{Antibodies and immunoblotting}

Western blot analysis was carried out as previously described (19). Briefly, cells were collected and washed with PBS, then lysed with RIPA buffer containing protease inhibitor (Sigma-Aldrich, St. Louis, MO, USA). After centrifugation, the supernatants were collected and quantified with BCA reagent. Equal amounts of protein $(25 \mu \mathrm{g})$ were separated on sodium dodecyl sulfate (SDS)polyacrylamide gel, and transferred onto a polyvinylidene difluoride (PVDF) membrane. After blocking with Trisbuffered saline containing $0.1 \%$ Tween-20 (SigmaAldrich, St. Louis, MO, USA) (TBST) and 5\% skimmed milk, membranes were probed with anti-LC3 (Abcam, Cambridge, MA, USA), anti-Beclin-1 (Abcam, Cambridge, MA, USA), anti-Atg5 (Abcam, Cambridge, MA, USA), anti-Bcl2 (Santa Cruz Biotechnology Inc., Santa Cruz, CA, USA), anti-Bax (Santa Cruz Biotechnology Inc., Santa Cruz, CA, USA), and anti-glyceraldehyde-3-phosphate dehydrogenase (GAPDH) (Cell Signaling Technology, Beverly, MA, USA) antibody in TBST containing 3\% nonfat milk. Afterwards, membranes were incubated with 

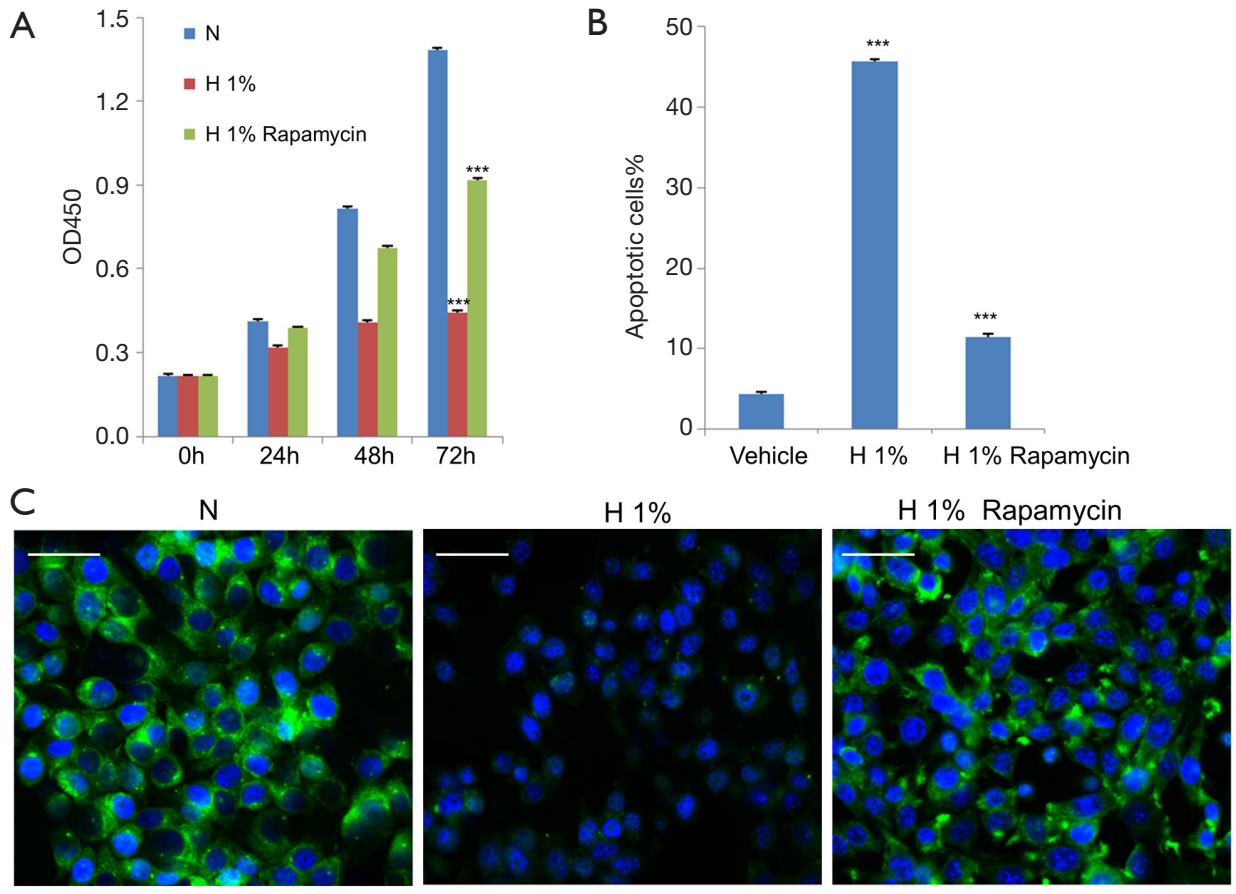

Figure 1 Hypoxia induced apoptosis and inhibited autophagy in D407 cells. (A) Cell growth was inhibited by hypoxia ( $\left.1 \% \mathrm{O}_{2}\right)$ over 72 h, and was partially recovered by the administration of rapamycin $(100 \mathrm{nM})$. (B) Hypoxia increased apoptosis compared to normoxia, which was inhibited by the addition of $100 \mathrm{nM}$ rapamycin. After having been subjected to hypoxia in the presence or absence of rapamycin for 48 h, human D407 RPE cells were collected and analyzed with fluorescence-activated cell sorting (FACS). (C) The autophagy marker LC3 was measured with fluorescence microscopy after treatment with hypoxia for 48 h. Hypoxic treatment decreased the immunofluorescence of LC3 (green), which was reversed by rapamycin (100 nM). The nuclei were stained with 4',6-diamidino-2-phenylindole (DAPI) in blue. Scale bar: $50 \mu \mathrm{m}$. Asterisks indicate significant difference $\left.{ }^{* * *}, \mathrm{P}<0.001\right)$.

horseradish-peroxidase-conjugated secondary antibodies.

\section{Statistical analysis}

All experiments were performed in triplicate and the results are provided as mean \pm SD. Data were analyzed using SPSS version 16.0 (SPSS Inc., IBM, Chicago, IL, USA). Statistical comparisons between means were carried out using student's $t$ test (two groups) or one-way ANOVA followed by Duncan's-test (more than two groups). Differences with $\mathrm{P}$ values $<0.05$ were considered statistically significant.

\section{Results}

\section{Hypoxia induced apoptosis and inbibited autophagy in d407 cells}

To specifically address the role of hypoxia in RPE function, we employed in vitro model of hypoxic insult with D407 cells according to Bellot et al.'s report. On this basis, we evaluated the individual effects on cell growth, apoptosis, and autophagy.

As shown in Figure $1 \mathrm{~A}$, cell growth was significantly and persistently attenuated under hypoxia within $72 \mathrm{~h}(\mathrm{P}<0.001)$, which followed a time-dependent manner. In terms of apoptosis, we detected the phosphatidylserine flipping with Annexin- $V$ binding assay (20) after hypoxic treatment for $48 \mathrm{~h}$. The flow cytometry results are shown in Figure $1 B$ and Figure S1. It was found that $1 \%$ oxygen was able to induce a large amount of apoptosis in $\mathrm{D} 407$ cells $(\mathrm{P}<0.001)$ when compared to control cells, which increased from $5 \%$ to more than $40 \%$. We also used immunofluorescence for endogenous LC3 to identify autophagosomes and monitored autophagy by fluorescence microscopy (21). As shown in Figure $1 C$, a significant decrease in the immunofluorescence signal of LC3 was observed in 1\% oxygen-administrated D407 cells, indicating the inhibition of autophagy.

To explore the potential role of autophagy in D407 


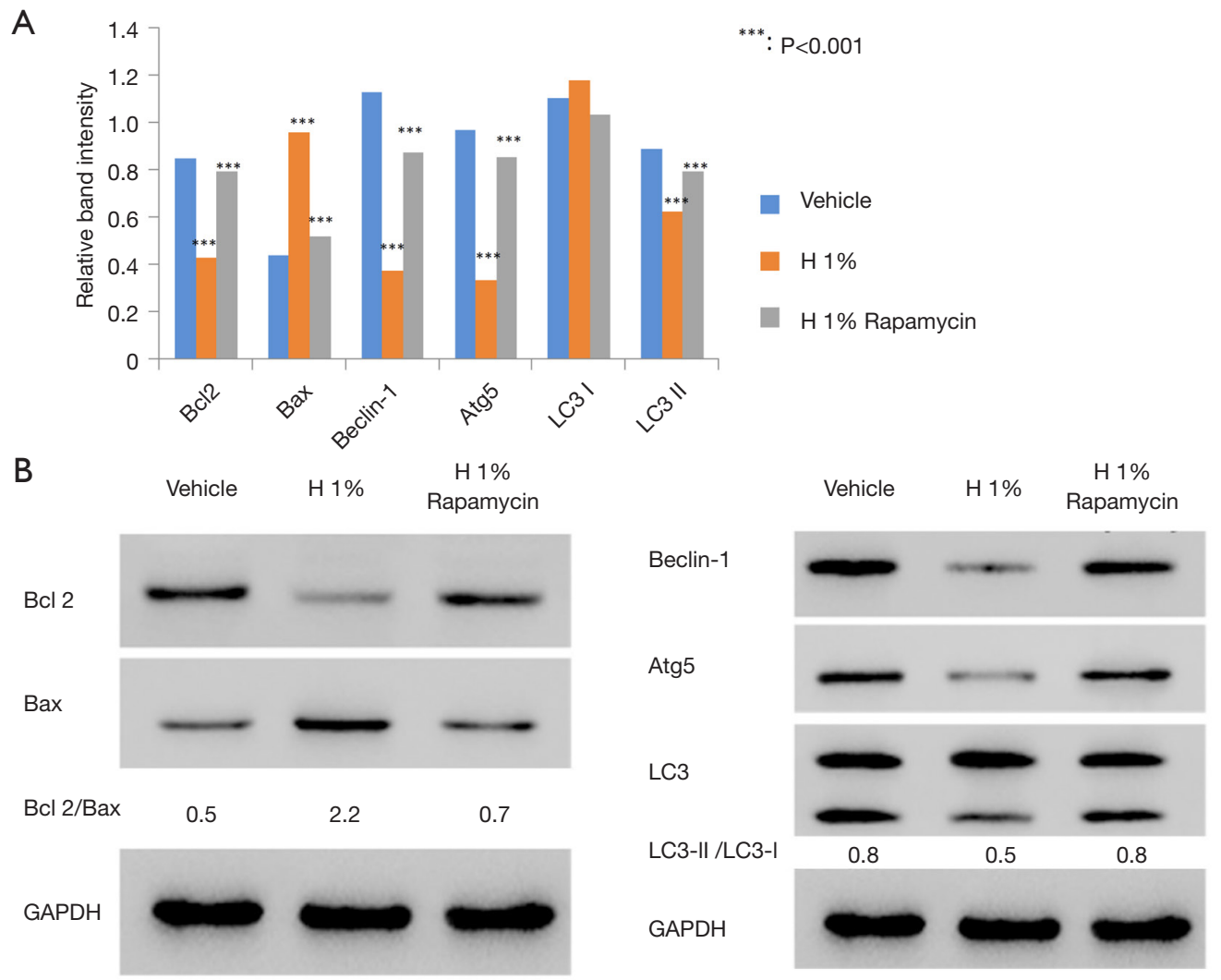

Figure 2 The expressions of apoptosis and autophagy associated proteins were investigated with western blotting. (A) The expression of Bcl2 decreased and Bax increased after hypoxic treatment for $48 \mathrm{~h}$. The administration of rapamycin (100 nM) reversed these effects in D407 cells. (B) The expressions of Beclin-1, Atg5, and the ratio of LC3-II/LC3-I decreased in hypoxia-treated D407 cells. The administration of rapamycin (100 $\mathrm{nM})$ reversed these effects in D407 cells. Asterisks indicate significant difference $(* * *, \mathrm{P}<0.001)$.

cells under hypoxic stress, we turned to the established autophagy inducer rapamycin, which is known to stimulate Beclin-1 dependent autophagy (7). We administrated D407 cells under $1 \%$ oxygen with $100 \mathrm{nM}$ rapamycin $(22,23)$ and found the pharmacological intervention significantly counteracted the hypoxic effects. Specifically, rapamycin alleviated the apoptosis (Figure $1 B$ ) and inhibition of cell growth that was induced by hypoxia (Figure 1A). As expected, the immunofluorescence signal of $\mathrm{LC} 3$, as the autophagy marker, was dramatically strengthened (Figure $1 C)$ which suggested that autophagy had been activated.

\section{Hypoxia increased Bax/Bcl-2 ratio and decreased LC3-II/I ratio in $\mathrm{D} 407$ cells}

To study the molecular basis underlying the abovementioned results, we examined the expression of regulatory proteins for both apoptosis (Bcl2, Bax) and autophagy (Beclin-1,
Atg5, LC3-I/II). According to western blot analysis, it was found that the hypoxic environment resulted in elevated expression of pro-apoptotic Bax and reduced production of anti-apoptotic Bcl-2 (Figure 2A). Further densitometric analysis revealed the significant increase of $\mathrm{Bax} / \mathrm{Bcl}-2$ ratio from 0.5 to 2.2 , which is an important indicator for the induction and processing of apoptosis (24).

We also examined the changes of autophagy-associated markers. The elevated LC3-II/LC3-I ratio is a hallmark of autophagy and correlates with the increased number of autophagosomes (25). Thus, we assessed the amounts of LC3-I (free cytosolic form) and LC3-II (autophagosomal membrane-bound form), as well as the conversion between them. Immunoblotting results showed that the ratio of LC3-II/LC3-I significantly decreased from 0.8 to 0.5 after $48 \mathrm{~h}$ of hypoxic treatment, suggesting the inhibition of endogenous LC3-II turnover in D407 cells (Figure 2B). This was consistent with the finding of autophagy 

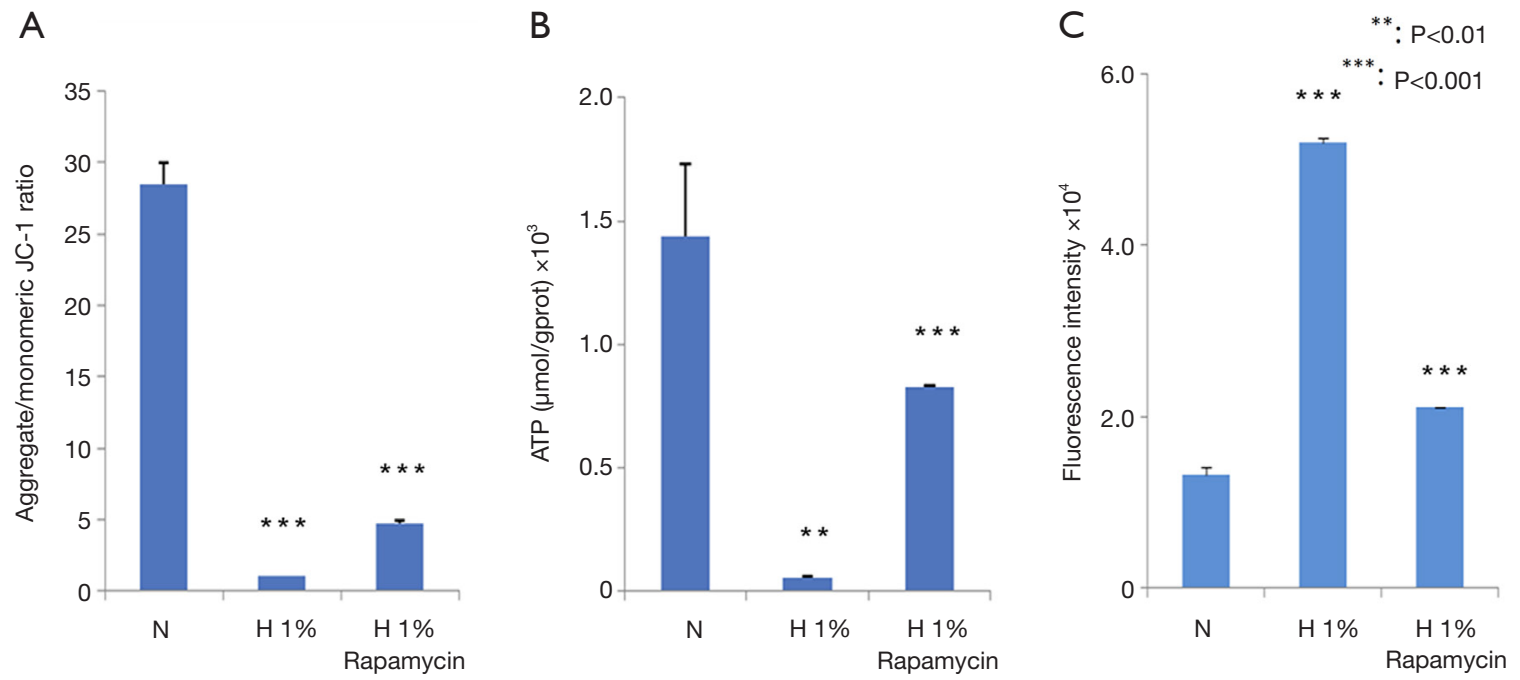

Figure 3 The mitochondrial function was impaired by hypoxia and partially recovered by the administration of $100 \mathrm{nM}$ rapamycin. (A) Mitochondrial membrane potential decreased in hypoxia-treated D407 cells. (B) The intracellular ATP concentration decreased in hypoxiatreated D407 cells. (C) Reactive oxygen species (ROS) level increased in hypoxia-treated D407 cells. Asterisks indicate significant difference $\left({ }^{* *}, \mathrm{P}<0.01 ;{ }^{* * *}, \mathrm{P}<0.001\right)$.

inhibition. In addition, we evaluated the difference in the expressions of Beclin-1 [an autophagy effector $(26,27)$ ] and Atg 5 [the basic autophagic machinery (28)]. In our results, the expressions of both proteins were significantly reduced by hypoxia (Figure $2 B$ ). On the other hand, the administration of rapamycin decreased the $\mathrm{Bax} / \mathrm{Bcl}-2$ ratio but increased the ratio of LC3-II/LC3-I and the expression of Beclin-1 and Atg5.

\section{Hypoxic effects on MMP, ROS and ATP production in D407 cells}

We further examined the changes in MMP, ATP, and ROS productions. Hypoxia decreased the MMP (Figure $3 A$ ), reduced the production of ATP (Figure $3 B$ ), and enhanced the generation of ROS (Figure 3C). All these changes were statistically significant $(\mathrm{P}<0.001)$, which suggested the dysfunction of mitochondria in hypoxia-treated D407 cells. In contrast, the administration of rapamycin counteracted these effects to a large extent $(\mathrm{P}<0.001)$.

\section{Overexpression of BNIP3 counteracted the bypoxic effects on D407 cells}

As the direct regulator of Beclin-1, BNIP3 is a major enabler of hypoxia-induced autophagy (14). Thus, we tried to study the potential role of BNIP3 in the context of hypoxic assaults on D407 cells. As expected, expression of BNIP3 decreased significantly with hypoxia treatment (Figure 4A). We then generated the lentiviral expression vector for BNIP3, with which we infected D407 cells. The increased expression level of BNIP3 was confirmed with western blotting (Figure 4B). On this basis, we examined the effects of BNIP3 overexpression on hypoxia-treated D407 cells. Interestingly, very similar results were obtained as those with the administration of rapamycin. Specifically, BNIP3 overexpression significantly promoted cell growth in a time-dependent manner (Figure 4C). Besides, it simultaneously inhibited hypoxia-induced apoptosis (Figure 4D) and activated autophagy (Figure 4E). Moreover, BNIP3 overexpression decreased the ratio of $\mathrm{Bax} / \mathrm{Bcl} 2$ from 2.3 to 0.7 but increased LC3-II/LC3-I ratio from 0.5 to 0.7. It also elevated the expressions of Beclin-1 and Atg5 (Figure 4F). In contrast, 3-MA, an established inhibitor of autophagy, was able to eliminate all these effects exerted by BNIP3 overexpression. Together, these results implied that BNIP3 be protective against hypoxia in human RPE cells through the mediation of autophagy.

\section{Effects of BNIP3 overexpression on MMP, ROS, and ATP production in hypoxia-treated D407 cells}

We assessed the effects of BNIP3 overexpression on the function of mitochondria. As shown in Figure 5, it largely 
A

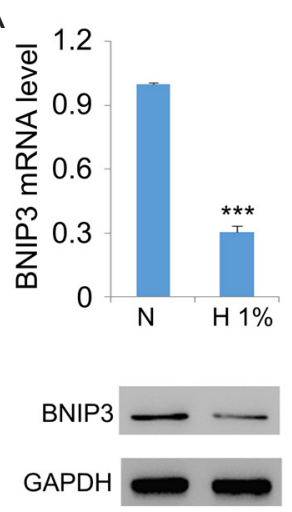

E

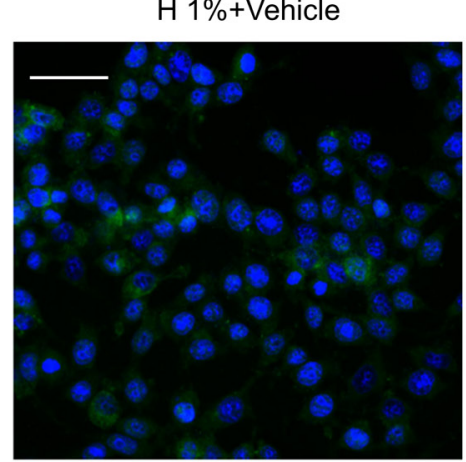

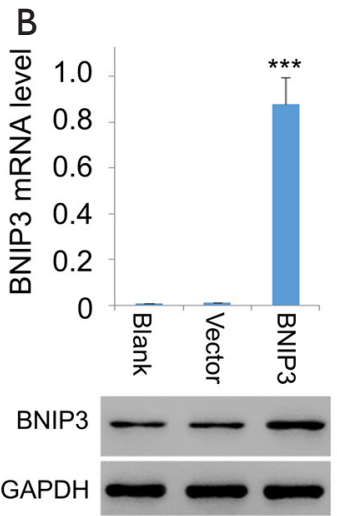

C

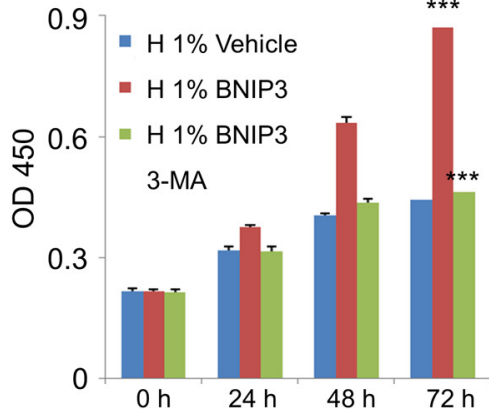

D

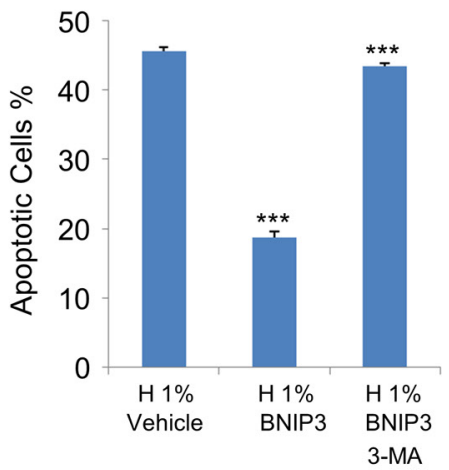

H $1 \%+B N I P 3$

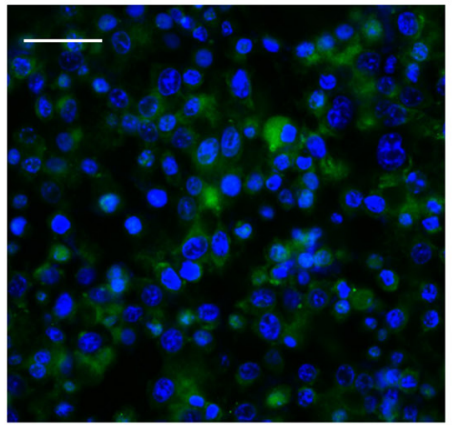

$\mathrm{H} 1 \%+\mathrm{BNIP} 3+3-\mathrm{MA}$

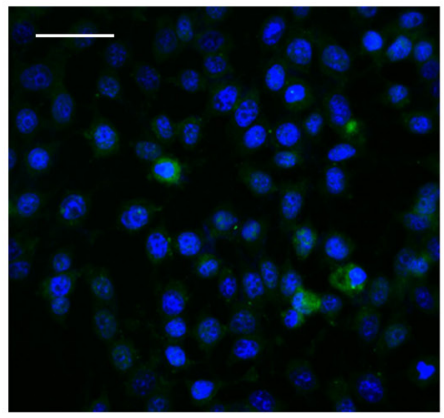

F

$$
\begin{array}{lll}
\text { H 1\% } & \text { H } 1 \% & \text { H 1\% } \\
\text { +Vehicle } & \text { +BNIP3 } & \text { +BNIP3+3-MA }
\end{array}
$$

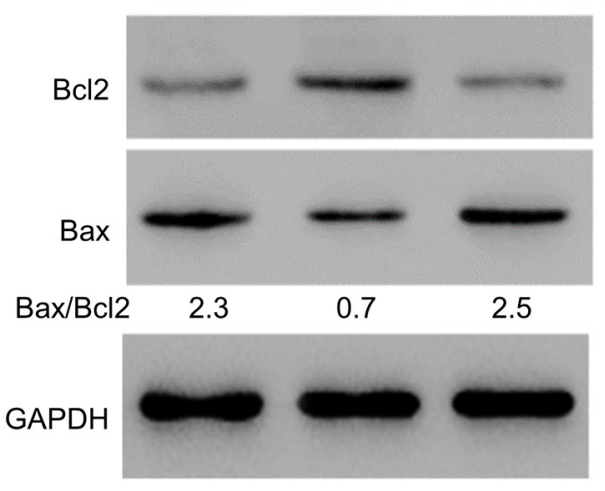

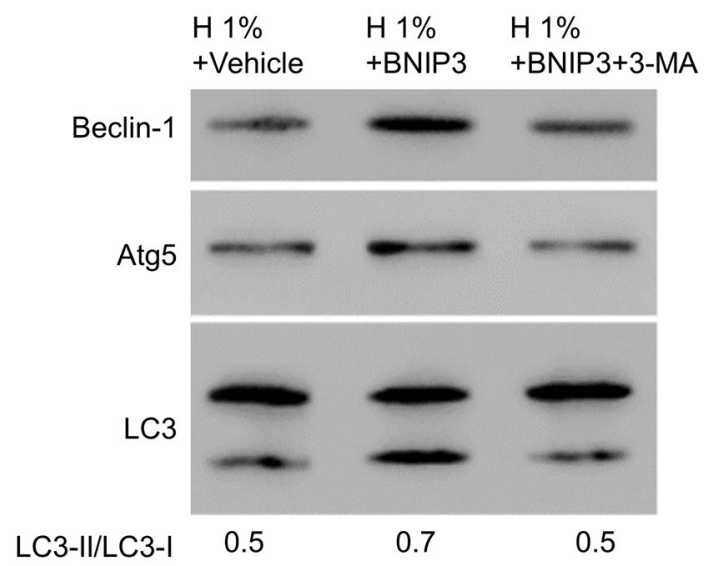

Figure 4 Overexpression of BNIP3 in D407 cells alleviated the impairments of hypoxia. (A) Decreased expression of BNIP3 was investigated with quantitative RT-PCR (top) and western blotting (bottom). Glyceraldehyde-3-phosphate dehydrogenase (GAPDH) was used as an internal control. (B) Quantitative RT-PCR (top) and western blotting (bottom) results to verify the overexpression of BNIP3 in D407 cells after infection with BNIP3 or lentivirus vector. GAPDH was used as an internal control. (C) BNIP3 overexpression promoted the cell growth in hypoxic conditions, which was inhibited by the administration of $50 \mu M$ 3-MA. (D) Hypoxia induced apoptosis in D407 cells, which was inhibited by BNIP3 overexpression. (E) Overexpression of BNIP3 recovered the inhibited autophagy by hypoxia to some extent. The immunofluorescence of LC3 (green) in D407 cells with or without BNIP3 overexpression was measured with fluorescence microscopy. The nuclei were stained with 4',6-diamidino-2-phenylindole (DAPI) in blue. Scale bar: $50 \mu \mathrm{m}$. (F) Overexpression of BNIP3 alleviated the impairments of hypoxia. Asterisks indicate significant difference $\left({ }^{* *}, \mathrm{P}<0.001\right)$. 
A

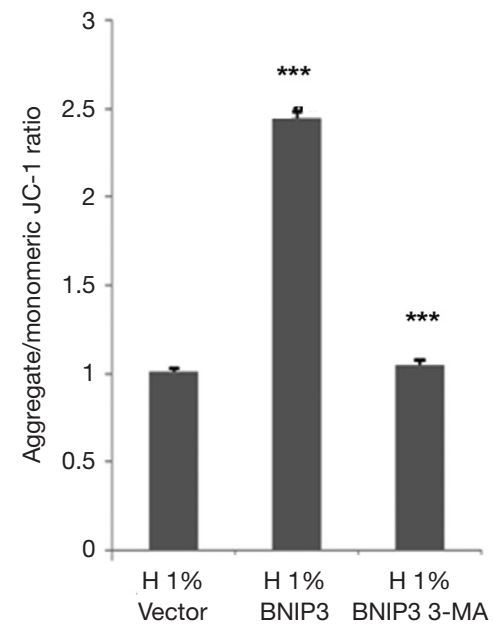

B

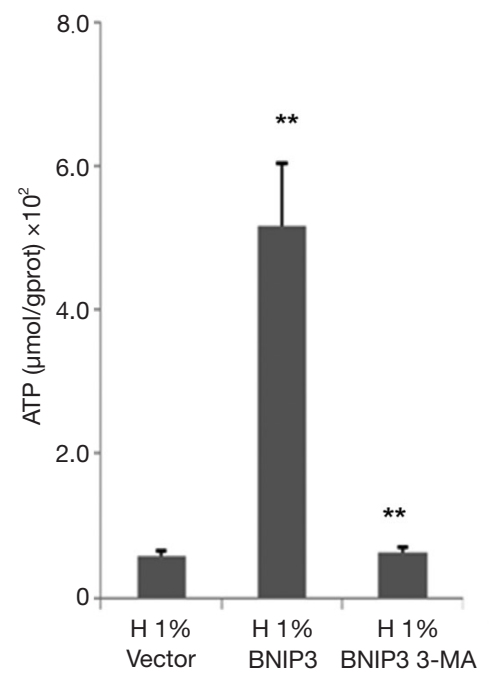

C

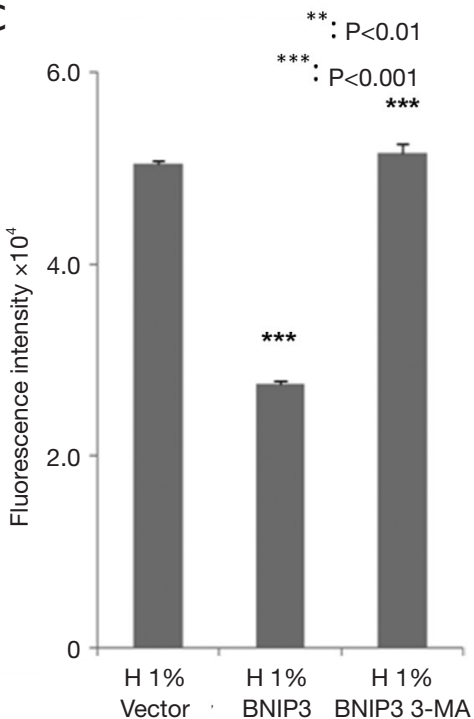

Figure 5 The hypoxic impairments in mitochondrial function were recovered by BNIP3 overexpression, which can be counteracted by 3-MA $(50 \mu \mathrm{M})$. (A) Mitochondrial membrane potential. (B) Intracellular ATP concentration. (C) Reactive oxygen species (ROS) level. D407 cells infected with BNIP3 or lentivirus vector were treated with hypoxia in the presence or absence of $50 \mu \mathrm{M} 3-\mathrm{MA}$ for $48 \mathrm{~h}$, then mitochondrial membrane potential, intracellular ATP concentration and ROS level were examined using different assays. Asterisks indicate significant difference $\left.{ }^{* *}, \mathrm{P}<0.01 ;{ }^{* * *}, \mathrm{P}<0.001\right)$.

recovered the MMP (Figure $5 A$ ), significantly increased the ATP production (Figure $5 B$ ), and lowered the ROS level (Figure 5C) in hypoxia-treated D407 cells. The administration of 3-MA eliminated these effects of BNIP3 overexpression almost completely.

\section{Knockdown of BNIP3 exaggerated hypoxic effects on D407 cells, which could be counteracted by rapamycin}

To construct the BNIP3-knockdown D407 cell line, we infected cells with manufactured shBNIP3 lentivirus and non-silencing shRNA lentivirus (scrambled). Afterwards, the expression of BNIP3 was measured by real-time qPCR and western blot analysis, respectively. Consistently, both mRNA and protein levels of BNIP3 were significantly decreased after shBNIP3 lentivirus infection compared to cells infected with scrambled shRNA lentivirus (Figure $6 A)$. Next, the effects of BNIP3 knockdown on cell survival were examined. As shown in our results, BNIP3 knockdown aggravated the hypoxic impairments to D407 cells, which meant stronger cell growth inhibition (Figure $6 B)$, more severe induction of apoptosis ( $>60 \%$, Figure $6 C$ ), and inhibition of autophagy (Figure 6D). In contrast, the administration of rapamycin, an acute inhibitor of mTOR complex 1 (mTORC1), was able to counteract these effects by BNIP3-knockdown to the full extent.

\section{Discussion}

Hypoxia in RPE cells may be a central risk factor for AMD (5). In the current study, $1 \%$ oxygen exposure significantly attenuated cell growth and induce a large amount of apoptosis in D407 cells, which is consistent with the knowledge that hypoxia plays an essential role in the impairments to RPE cells (5). Interestingly, the above findings disagreed with Bellot et al.'s report that $1 \%$ oxygen treatment generally contributed to survival rather than cell death among different types of cells (14). In our opinion, it could be attributed to the fact that different severity of hypoxia determines different cellular response to the stress (29). Additionally, difference in cell types may also account for the variance in performance under similar circumstances.

According to previous reports, acute exposure to hypoxia resulted in an acute increase in ROS generation by complex III of the mitochondrial electron transport chain; the failure to properly regulate mitochondrial metabolism in response to hypoxia was associated with increased ROS levels and 


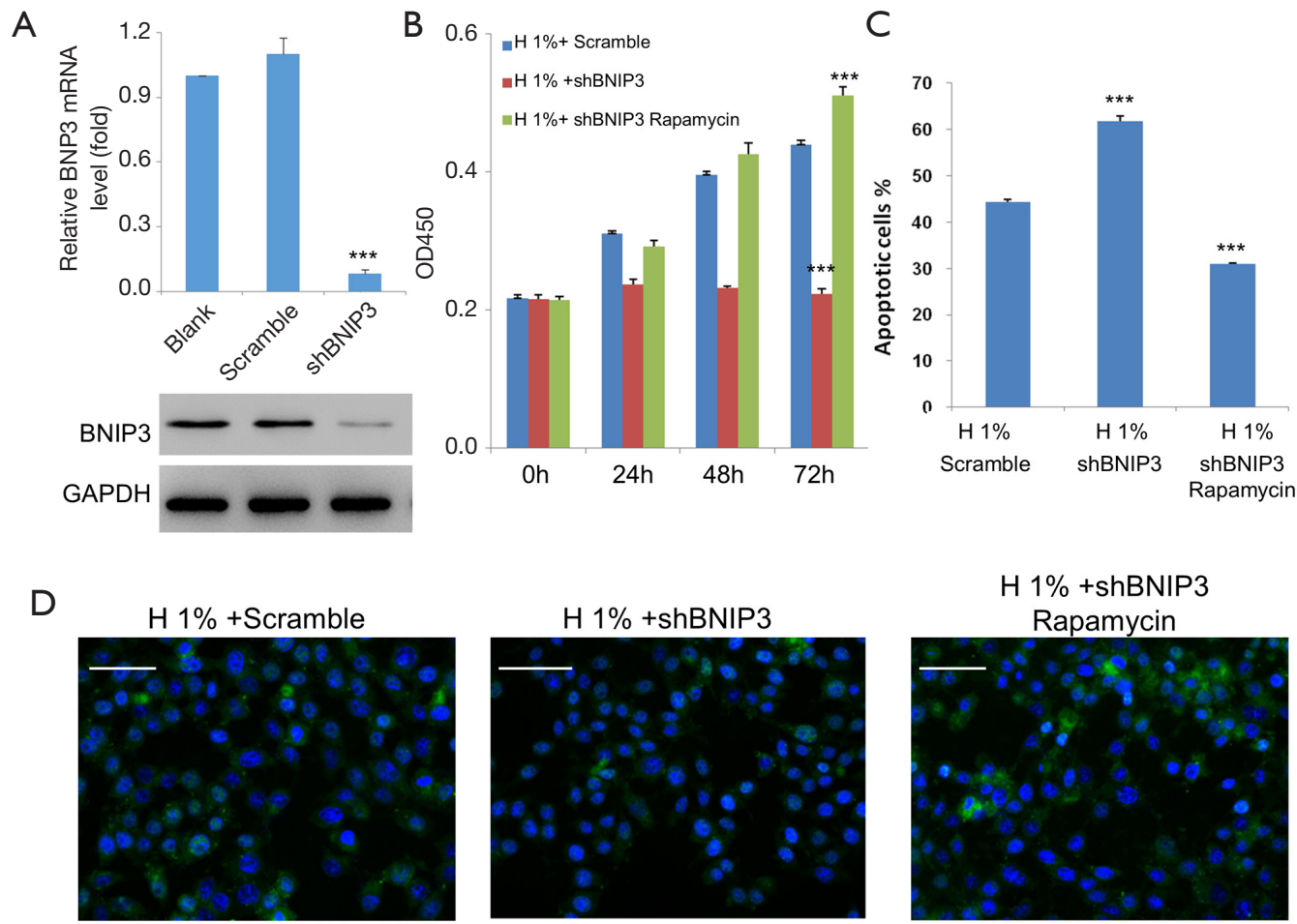

Figure 6 The hypoxic impairments to D407 cells were further aggravated by BNIP3 knockdown, which was alleviated by the administration of $100 \mathrm{nM}$ rapamycin. (A) Quantitative RT-PCR (top) and western blot analysis (bottom) of BNIP3 expressions in D407 cells after infection with BNIP3 shRNA (shBNIP3) or control shRNA lentivirus (scrambled). Glyceraldehyde-3-phosphate dehydrogenase (GAPDH) was used as an internal control. (B) Knockdown of BNIP3 further inhibited the cell growth under hypoxic conditions. (C) The number of apoptotic cells was increased by BNIP3 knockdown. (D) LC3 immunofluorescence signals (green) were diminished by BNIP3 knockdown. The nuclei are stained with 4',6-diamidino-2-phenylindole (DAPI) in blue. Scale bar: $50 \mu \mathrm{m}$. Asterisks indicate significant difference $\left.{ }^{* * *}, \mathrm{P}<0.001\right)$.

increased cell death. On the other hand, mitochondrial autophagy is an adaptive metabolic response to hypoxia and could decrease mitochondria damage under hypoxia (12). In terms of RPE cells, Mitter et al. found that oxidative stress, which is linked to hypoxia in AMD, can result in dysregulation of autophagy (13). The protein LC3-II is an important marker of autophagy (30) and autophagy could be assessed by determining the lysosomal turnover of microtubule-associated protein 1 light chain 3 (LC3)-II (31). In the current study, 1\% oxygen exposure significantly inhibited endogenous LC3-II turnover in D407 cells. Previously, Bellot et al. demonstrated that autophagy induction contributed to survival rather than cell death of various cell types under sub-lethal hypoxic conditions (14). These seemingly contradictory findings suggest that hypoxia may regulate autophagy and cell survival in a context-dependent manner.

Moreover, the elevated $\mathrm{Bax} / \mathrm{Bcl}-2$ ratio generally corresponds with the onset of cell apoptosis (32). When autophagy is activated, the $18 \mathrm{kDa} \mathrm{LC} 3-\mathrm{I}$ protein which is localized in the cytoplasm can be converted to $16 \mathrm{kDa}$ LC3-II which inserts into autophagosome membranes (26). The elevated LC3-II/LC3-I ratio is a hallmark of autophagy and correlates with the increased number of autophagosomes (28). The protein $\operatorname{Atg} 5$, as the basic autophagic machinery, is involved in the early stages of autophagosome formation (28). Beclin-1, as the autophagy effector, is required for the Atg5-dependent autophagy and responsible for autophagic vesicle nucleation in mammals $(26,27)$. The ablation of Beclin-1 or Atg5 would enhance cell death under hypoxic conditions (14). Here, the administration of autophagy inducer rapamycin decreased the Bax/Bcl-2 ratio but increased the ratio of LC3-II/ LC3-I and the expression of Beclin-1 and Atg5. These findings agreed with the report by Wang et al. that showed rapamycin administration significantly increased the protein 
expression levels of LC3-II and Beclin-1 (33). Rapamycin was able to stimulate autophagy and protected RPE cells from hypoxia, which implied the protective role of autophagy against hypoxia in human RPE cells. Our results confirmed that increased autophagy induction could protect human RPE cells against hypoxic impairments.

Bellot et al. has reported that BNIP3 plays an essential role in hypoxia-induced mitochondrial autophagy (14). The present study showed that BNIP3 overexpression stimulate autophagy and counteract hypoxia-induced apoptosis. According to a previous report, 3-MA inhibits autophagy by down-regulating the expression of LC3 and Beclin-1 (34), which was also observed in our results. Interesting, 3-MA counteract the effects of BNIP3 overexpression. BNIP3 knockdown was observed to aggravate the hypoxic impairments to D407 cells. In contrast, the administration of rapamycin, an acute inhibitor of mTOR complex 1 (mTORC1), was able to counteract these effects by BNIP3knockdown to the full extent. Collectively, these data suggested that BNIP3 protected RPE cells from hypoxiainduced damage by inducing autophagy. However, given the multiple downstream signaling of mTORC1, we could not rule out the possible involvement of other downstream signaling of mTORC1, such as translation and metabolism. Thus, further elaboration is required before a conclusion can be made.

\section{Conclusions}

We found hypoxia induced massive apoptosis and inhibited autophagy simultaneously in the D407 cells. Moreover, hypoxia impaired the mitochondrial function by lowering membrane potential, reducing ATP production, and increasing ROS level. Interestingly, the administration of rapamycin was able to stimulate autophagy and counteract the above effects of hypoxia to a large extent, which implied the protective role of autophagy against hypoxia in human RPE cells. More importantly, similar results were observed for BNIP3 overexpression, which can be largely blocked by the established inhibitor of autophagy 3-MA. Furthermore, BNIP3 knockdown was observed to aggravate the hypoxic impairments to D407 cells, which was greatly blocked by the administration of rapamycin. Altogether, we can conclude that BNIP3 protects human retinal pigmented epithelial cells under hypoxic conditions, possibly by inducing autophagy. Hypoxia in RPE cells may play a central role for the pathology of AMD, thus BNIP3 may be a possible target in the future development of target for novel therapies against AMD. Consider the complex environment in vivo, animal experiments should be carried out to further verify the results of cell experiments in the future.

\section{Acknowledgments}

We express gratitude to Qing Gu for generously providing the human RPE D407 cell line. We would like to thank Xueting Luo for expert assistance in flow cytometry.

Funding: This study was supported by the Shanghai Pujiang Program (16PJ1408500), the National Science Fund for Distinguished Young Scholars of China (81425006), and the National Natural Science Foundation for Young Scholars of China (81500732).

\section{Footnote}

Reporting Checklist: The authors have completed the MDAR reporting checklist. Available at http://dx.doi.org/10.21037/ atm-20-7145

Data Sharing Statement: Available at http://dx.doi. org/10.21037/atm-20-7145

Conflicts of Interest: All authors have completed the ICMJE uniform disclosure form (available at http://dx.doi. org/10.21037/atm-20-7145). The authors have no conflicts of interest to declare.

Ethical Statement: The authors are accountable for all aspects of the work in ensuring that questions related to the accuracy or integrity of any part of the work are appropriately investigated and resolved.

Open Access Statement: This is an Open Access article distributed in accordance with the Creative Commons Attribution-NonCommercial-NoDerivs 4.0 International License (CC BY-NC-ND 4.0), which permits the noncommercial replication and distribution of the article with the strict proviso that no changes or edits are made and the original work is properly cited (including links to both the formal publication through the relevant DOI and the license). See: https://creativecommons.org/licenses/by-nc-nd/4.0/.

\section{References}

1. Birch DG, Liang FQ. Age-related macular degeneration: 
a target for nanotechnology derived medicines. Int J Nanomedicine 2007;2:65-77.

2. Chen M, Chan CC, Xu H. Cholesterol homeostasis, macrophage malfunction and age-related macular degeneration. Ann Transl Med 2018;6:S55.

3. Strauss O. The retinal pigment epithelium in visual function. Physiol Rev 2005;85:845-81.

4. Telegina DV, Kozhevnikova OS, Bayborodin SI, et al. Contributions of age-related alterations of the retinal pigment epithelium and of glia to the AMD-like pathology in OXYS rats. Sci Rep 2017;7:41533.

5. Kurihara T, Westenskow PD, Gantner ML, et al. Hypoxiainduced metabolic stress in retinal pigment epithelial cells is sufficient to induce photoreceptor degeneration. Elife 2016;5:e14319.

6. Kinnunen K, Petrovski G, Moe MC, et al. Molecular mechanisms of retinal pigment epithelium damage and development of age-related macular degeneration. Acta Ophthalmol 2012;90:299-309.

7. Ravikumar B, Sarkar S, Davies JE, et al. Regulation of mammalian autophagy in physiology and pathophysiology. Physiol Rev 2010;90:1383-435.

8. Blokhina O, Virolainen E, Fagerstedt KV. Antioxidants, oxidative damage and oxygen deprivation stress: a review. Ann Bot 2003;91:179-94.

9. Blasiak J, Petrovski G, Veréb Z, et al. Oxidative stress, hypoxia, and autophagy in the neovascular processes of age-related macular degeneration. Biomed Res Int 2014;2014:768026.

10. Rickman CB, Farsiu S, Toth CA, et al. Dry age-related macular degeneration: mechanisms, therapeutic targets, and imaging. Invest Ophthalmol Vis 2013;54:ORSF68ORSF80.

11. Mazure NM, Pouysségur J. Hypoxia-induced autophagy: cell death or cell survival? Curr Opin Cell Biol 2010;22:177-80.

12. Zhang H, Bosch-Marce M, Shimoda LA, et al. Mitochondrial autophagy is an HIF-1-dependent adaptive metabolic response to hypoxia. J Biol Chem 2008;283:10892-903.

13. Mitter SK, Song C, Qi X, et al. Dysregulated autophagy in the RPE is associated with increased susceptibility to oxidative stress and AMD. Autophagy 2014;10:1989-2005.

14. Bellot G, Garcia-Medina R, Gounon P, et al. Hypoxiainduced autophagy is mediated through hypoxia-inducible factor induction of BNIP3 and BNIP3L via their BH3 domains. Mol Cell Biol 2009;29:2570-81.

15. He C, Klionsky DJ. Regulation mechanisms and signaling pathways of autophagy. Annu Rev Genet 2009;43:67-93.

16. Zufferey R, Nagy D, Mandel RJ, et al. Multiply attenuated lentiviral vector achieves efficient gene delivery in vivo. Nat Biotechnol 1997;15:871-5.

17. Lau A, Zheng Y, Tao S, et al. Arsenic inhibits autophagic flux, activating the Nrf2-Keap1 pathway in a p62dependent manner. Mol Cell Biol 2013;33:2436-46.

18. Zhang Y, Soboloff J, Zhu Z, et al. Inhibition of Ca2+ influx is required for mitochondrial reactive oxygen speciesinduced endoplasmic reticulum $\mathrm{Ca} 2+$ depletion and cell death in leukemia cells. Mol Pharmacol 2006;70:1424-34.

19. Szatmári-Tóth M, Kristóf E, Veréb Z, et al. Clearance of autophagy-associated dying retinal pigment epithelial cells - a possible source for inflammation in age-related macular degeneration. Cell Death Dis 2016;7:e2367.

20. van Engeland M, Nieland LJ, Ramaekers FC, et al. Annexin $\mathrm{V}$-affinity assay: a review on an apoptosis detection system based on phosphatidylserine exposure. Cytometry 1998;31:1-9.

21. Sharifi MN, Mowers EE, Drake LE, et al. Measuring autophagy in stressed cells. Stress Responses. Springer; 2015:129-50.

22. Sotthibundhu A, McDonagh K, von Kriegsheim A, et al. Rapamycin regulates autophagy and cell adhesion in induced pluripotent stem cells. Stem Cell Res Ther 2016;7:166.

23. Rezabakhsh A, Ahmadi M, Khaksar M, et al. Rapamycin inhibits oxidative/nitrosative stress and enhances angiogenesis in high glucose-treated human umbilical vein endothelial cells: role of autophagy. Biomed Pharmacother 2017;93:885-94.

24. Kim ME, Ha TK, Yoon JH, et al. Myricetin induces cell death of human colon cancer cells via BAX/BCL2dependent pathway. Anticancer Res 2014;34:701-6.

25. Kabeya Y, Mizushima N, Ueno T, et al. LC3, a mammalian homologue of yeast Apg8p, is localized in autophagosome membranes after processing. EMBO J 2000;19:5720-8.

26. Codogno P, Meijer AJ. Atg5: more than an autophagy factor. Nat Cell Biol 2006;8:1045-7.

27. Sinha S, Levine B. The autophagy effector Beclin 1: a novel BH3-only protein. Oncogene 2008;27:S137-48.

28. DeSelm CJ, Miller BC, Zou W, et al. Autophagy proteins regulate the secretory component of osteoclastic bone resorption. Dev Cell 2011;21:966-74.

29. Greijer AE, van der Wall E. The role of hypoxia inducible factor 1 (HIF-1) in hypoxia induced apoptosis. J Clin Pathol 2004;57:1009-14.

30. Kang R, Zeh H, Lotze M, et al. The Beclin 1 network 
regulates autophagy and apoptosis. Cell Death Differ 2011;18:571-80.

31. Tanida I, Ueno T, Kominami E. LC3 and Autophagy. Autophagosome and Phagosome. Springer; 2008:77-88.

32. Perlman H, Zhang X, Chen MW, et al. An elevated bax/ bcl-2 ratio corresponds with the onset of prostate epithelial cell apoptosis. Cell Death Differ 1999;6:48-54.

33. Wang Y, Ma Q, Ma X, et al. Role of mammalian target of rapamycin signaling in autophagy and the

Cite this article as: Feng J, Tan W, Li T, Yan Q, Zhu H, Sun X. Human retinal pigment epithelial cells are protected against hypoxia by BNIP3. Ann Transl Med 2020;8(22):1502. doi: $10.21037 /$ atm-20-7145 neurodegenerative process using a senescence accelerated mouse-prone 8 model. Exp Ther Med 2017;14:1051-7.

34. Ni H, Gong Y, Yan JZ, et al. Autophagy inhibitor 3-methyladenine regulates the expression of LC3, Beclin-1 and $\mathrm{ZnTs}$ in rat cerebral cortex following recurrent neonatal seizures. World J Emerg Med 2010;1:216-23.

(English Language Editor: J. Jones) 